\title{
Respuesta del crecimiento de Pinus oocarpa a variables climáticas en Chiapas, México
}

\author{
María I. López-Hernández ${ }^{1}$, Julián Cerano-Paredes ${ }^{2 *}$, Salvador Valencia-Manzo ${ }^{1}$, \\ Eladio H. Cornejo-Oviedo ${ }^{1}$, José Villanueva-Díaz ${ }^{2}$, Rosalinda Cervantes-Martínez ${ }^{2}$ \\ \& Gerardo Esquivel-Arriaga ${ }^{2}$ \\ 1. Departamento Forestal, Universidad Autónoma Agraria Antonio Narro, Calzada Antonio Narro No 1923, Buenavista, \\ CP 25315, Saltillo, Coahuila; mary_isa_18@hotmail.com, svalenciam2016@gmail.com, e.cornejo@forestal.org.mx \\ 2. Laboratorio de Dendrocronología, INIFAP CENID-RASPA, km 6.5 Margen derecha del canal Sacramento, CP 35140 , \\ Gómez Palacio, Durango; cerano.julian@gmail.com,*villanueva.jose@inifap.gob.mx, \\ rosy_cervantes23@yahoo.com.mx, esquivel.gerardo@inifap.gob.mx \\ * Correspondencia
}

Recibido 07-III-2018. C Corregido 03-VIII-2018. Aceptado 03-IX-2018.

\begin{abstract}
Growth response of Pinus oocarpa to climatic variables in Chiapas, Mexico. Dendrochronological studies are used to reconstruct some climatic variables; in México these studies have focused on central and Northern temperate forests where trees present well defined annual rings. Few studies have been carried out in the Southern part of the country where annual ring growth is not easily identified and thus makes cross-dating problematic. We analyzed the dendrochronological potential of Pinus oocarpa Schiede for reconstructing climatic variables in the Northwest portion of Lagunas de Montebello, Chiapas. We used a selective sampling approach and collected 65 increment cores from 34 trees. While our samples showed a high frequency of false rings ( 8 to $60 \%$ ), we were able to date 30 samples from 22 trees (46\%) using standard dendrochronological techniques and developed total chronologies for total ring width, earlywood, and latewood for a period of 91 years (1925-2015). We found a significant influence of mean precipitation and mean maximum and minimum temperature over the annual ring growth of $P$. oocarpa in the period 1961-2004. Our results show that winter-spring precipitation (January-May) was the most important for the species' annual ring growth. However, we found the highest correlation between spring (March-May) precipitation and the earlywood chronology $(r=0.719, \mathrm{P}<0.05)$. The earlywood chronology also showed potential for reconstructing minimum temperatures (March to May) ( $\mathrm{r}=$ $0.732, \mathrm{P}<0.05)$, while the latewood chronology had the potential for reconstructing the maximum temperature (September to January) $(\mathrm{r}=0.714, \mathrm{P}<0.05)$. These results showed that $P$. oocarpa can be used to reconstruct climatic variables in the Mexican tropics. We recommend that new areas with older trees should be explored in order to increase the depth of chronologies and reconstruct climate records several centuries into the past.
\end{abstract}

Key words: growth rings; false rings; earlywood; latewood; dendroclimatic potential.

López-Hernández, M. I., Cerano-Paredes, J., Valencia-Manzo, S., Cornejo-Oviedo,E. H., Villanueva-Díaz, J., Cervantes-Martínez, R., \& Esquivel-Arriaga, G. (2018). Respuesta del crecimiento de Pinus oocarpa a variables climáticas en Chiapas, México. Revista de Biología Tropical, 66(4), 1580-1596.

Ante el actual proceso de cambio en las condiciones del clima, existe el interés científico a nivel mundial por estudiar la variabilidad climática en el pasado, para esto, se han empleado diversos tipos de registros proxy; entre los más importantes se encuentran los sedimentos de lagos, hielo, corales, archivos históricos, análisis de polen y anillos de crecimiento de árboles (Bradley, 1999). Para este último, la disciplina que se encarga de 
interpretar dichos registros, se conoce como dendrocronología (Benito, 2014), que se define como el estudio de los anillos de crecimiento de los árboles para fechar eventos pasados (Stokes \& Smiley, 1996).

El crecimiento de los árboles en regiones tropicales es casi continuo, lo que hace que se dificulte distinguir bandas o anillos de crecimiento anual; las coníferas en estas latitudes además desarrollan anillos falsos, lo que incrementa el problema para definir anillos anuales, a lo cual se atribuye que existan pocos estudios dendrocronológicos es estas regiones (Hastenrath, 1963; Johnson, 1980; Szenjner, 2011). Aunque las investigaciones dendrocronológicas en el trópico se remontan a más de 100 años (Worbes, 2002), algunos científicos dudan de la capacidad de los árboles tropicales para formar anillos de crecimiento anual (Turner, 2004). En 1870 se realizó el primer estudio dendrocronológico en el trópico, estudiando el incremento radial de la teca (Tectona grandis Lf.), en la cual se determinó los ciclos de corta con base en los anillos de crecimiento; de este modo, se estableció un sistema silvícola sostenible (Worbes, 2002). Posteriormente, se estudió Cordia alliodora (Ruíz y Pav.) Oken en América tropical, este estudio fue desarrollado por César Pérez en 1954 (Tschinkel, 1966) quien supuso acertadamente que los anillos eran anuales.

Recientemente, se han realizado estudios dendrocronológicos con el género Pinus, como el de Johnson (1980) que estudió P. oocarpa en Honduras, no obstante que, reporta la formación de anillos falsos, logró extender la red de cronologías de anillos de árboles para el trópico. Szenjner (2011) estudio P. oocarpa en la región oriental de Guatemala, donde analizó la anatomía del anillo y factores que influyen en el crecimiento de esta especie, logrando extender el conocimiento del clima en esta región.

Para México y Centroamérica, se reportan 47 especies de Pinus (Perry, Graham, \& Richardson, 1998). En el norte y centro de México se ha explorado el potencial dendroclimático de varias coníferas como Pseudotsuga menziesii (Mirb.) Franco (Villanueva, Fulé, Cerano, Estrada, \& Sánchez, 2009; Cerano et al., 2011), Pinus cooperi Blanco, Pinus durangensis Martínez, Pinus lumholtzii Rob. et Fern. (Chavez et al., 2017), Abies religiosa (Cerano et al., 2014) y Pinus hartwegii Lindl. (Villanueva et al., 2015). Para Centroamérica, Hastenrath (1963), Johnson (1980) y Szenjner (2011), han realizado estudios analizando los crecimientos de P. oocarpa.

En México, $P$. oocarpa registra una distribución desde el norte hasta el sur del país (Martínez, 1992). A pesar de su amplia distribución, sólo existen un par de referencias sobre el análisis dendroclimático de esta especie, la primera realizada en la región de Los Tuxtlas, Veracruz (Gutiérrez, 2013) y la segunda en el bosque La Primavera en Jalisco (Villanueva et al., 2018). Estudios dendroclimáticos en el sur del país con base en P. oocarpa, son muy limitados. Esta especie puede representar una alternativa para reconstruir información climática en zonas del trópico mexicano, donde se carece de datos climáticos instrumentales extensos; por tal motivo, la presente investigación tiene como objetivos: 1) desarrollar una cronología con base en los anillos de crecimiento de $P$. oocarpa para la porción noroeste de las Lagunas de Montebello, Chiapas, y 2) determinar el potencial dendroclimático de $P$. oocarpa para reconstruir variables climáticas en esta región del trópico mexicano. Con base en los resultados de los estudios desarrollados en Centroamérica por Hastenrath (1963), Johnson (1980) y Szenjner (2011) y de México (Gutiérrez, 2013), que reportan problemas en el crecimiento de $P$. oocarpa para su fechado; en este estudio se plantean dos preguntas de investigación: 1) ¿A pesar de los problemas de crecimiento documentados para esta especie, es factible generar una cronología para esta región del trópico mexicano? y 2) ¿El crecimiento anual de $P$. oocarpa es sensible a la variabilidad climática que experimenta esta región de México?

\section{MATERIALES Y MÉTODOS}

Área de estudio y muestreo: Se localiza en el Ejido Ojo de Agua, La Independencia, 
Chiapas (16 09' 24" N - 91 $45^{\circ} 29^{\circ}$ ' O), a una altitud de $1527 \mathrm{msnm}$ (Fig. 1) (INEGI-CONAGUA, 2007). La región hidrológica la conforman los ríos Grijalva-Usumacinta, el origen de las rocas es sedimentario (INEGI, 2005). Los suelos predominantes son litosoles (INEGI, 2007). El clima es templado subhúmedo con una temperatura media de 12 a $18^{\circ} \mathrm{C}$ y una precipitación anual de 1500 mm (García, 1998). La vegetación presente es secundaria arbustiva, bosque de pino-encino, bosque mesófilo de montaña y bosque de pino (Rzedowski, 1978).

Durante septiembre y noviembre 2016, se tomaron muestras de $P$. oocarpa empleando un muestreo selectivo. Se seleccionaron 34 árboles de los especímenes más longevos, libres de daños mecánicos, plagas y enfermedades. A cada árbol seleccionado, se le extrajo dos virutas (núcleos de crecimiento), en su mayoría a $1.30 \mathrm{~m}$ sobre el nivel del suelo con taladro de Pressler, se obtuvo un total de 65 núcleos de crecimiento (incrementos radiales).

Análisis de laboratorio: Las muestras se prepararon para su análisis en el Laboratorio de Dendrocronología del CENID-RASPA,
INIFAP, Gómez Palacio, Durango, México. Cada muestra fue montada en una base de madera para dar mayor firmeza y resistencia a las muestras. Una vez que se pegaron firmemente, se procedió a realizar un lijado para resaltar las estructuras de crecimiento, utilizando lijas de diferente grano (120-1200). Se realizó un pre-fechado de los anillos de crecimiento anual mediante gráficos de crecimiento (Skeleton plots) (Stokes \& Smiley, 1996). Las muestras de $P$. oocarpa presentaron alta frecuencia de anillos dobles o falsos, para distinguir estos anillos, se compararon patrones de crecimiento entre muestras del mismo árbol y posteriormente entre árboles, siguiendo las sugerencias de Stokes y Smiley (1996). Realizado el pre-fechado, se midió el crecimiento total anual, longitud de madera temprana y de madera tardía de cada una de las muestras, empleando un sistema de medición Velmex de $0.001 \mathrm{~mm}$ de precisión.

Análisis estadístico: La calidad del fechado de las series obtenidas (anillo total, madera temprana y madera tardía), se verificó con el programa COFECHA (Holmes, 1983), el cual estandariza cada serie y analiza

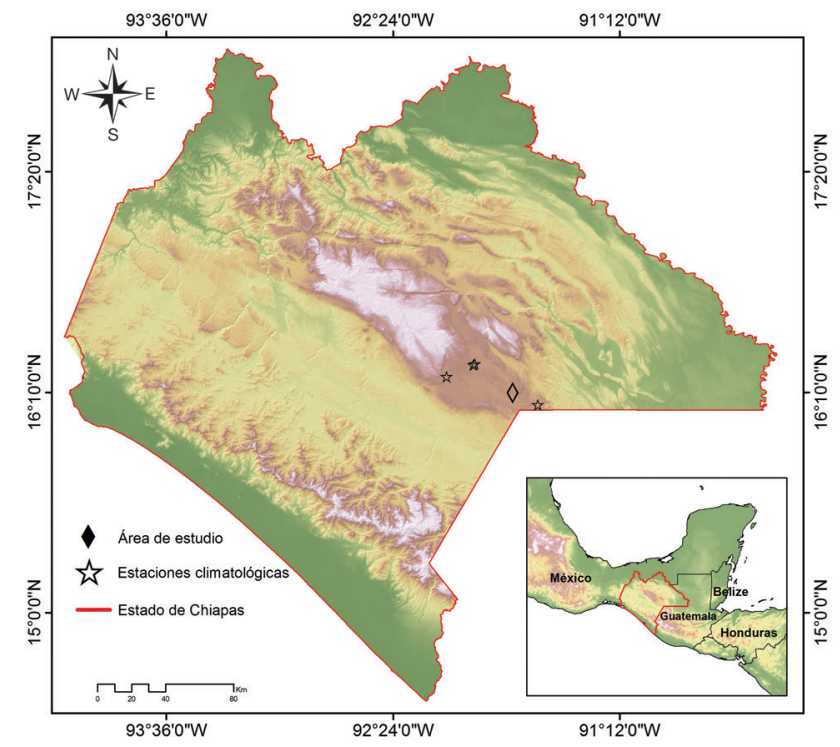

Fig. 1. Área de muestreo de Pinus oocarpa en el Ejido Ojo de Agua, La Independencia, Chiapas, y las estaciones climatológicas consideradas para el análisis dendroclimático.

Fig. 1. Sampling area of Pinus oocarpa at the Ejido Ojo de Agua, La Independencia, Chiapas and climatological stations considered for the dendroclimate analyzis. 
estadísticamente el fechado al correlacionar sucesivamente segmentos de 50 años con traslapes de 25 años, compara cada serie individual con todas las muestras y con la serie promedio; con base en este procedimiento, se identifican errores en el fechado atribuidos a la formación de anillos falsos o la no formación de anillos (anillos ausentes) en años particulares.

Fechado cada crecimiento, con el programa ARSTAN se generaron las cronologías o índices de anillo total, madera temprana y madera tardía. Se aplicó la mejor curva de ajuste (exponencial negativa, lineal, entre otras) a cada serie de crecimiento (Cook, 1987), para remover la varianza debido a factores biológicos (competencia y liberación) y geométricos (el área de fuste se incrementa con la edad y el crecimiento anual tiende a disminuir al distribuirse en una mayor superficie) no relacionados con el clima y maximizar la varianza debida a factores ambientales (factores climáticos y atmosféricos) que afectan la población. $\mathrm{Al}$ dividir el valor del ancho del anillo entre el valor correspondiente de la curva, se obtuvo el índice de crecimiento para cada año. Finalmente, al promediar los índices anuales de las series individuales, se generó la cronología del sitio. El programa ARSTAN produce tres cronologías, estándar, residual y arstan, para el presente estudio, se trabajó con la cronología estándar por conservar la variación de media y alta frecuencia y registrar los mejores resultados al relacionarla con variables climáticas.

El potencial de $P$. oocarpa para registrar la variabilidad climática regional, se determinó mediante un análisis de función de respuesta, para lo cual, se relacionaron los datos promedio mensuales regionales (44 años: 1961-2004), tanto de precipitación (estaciones Comitán, Margaritas, Santa Elena y Tziscao) como de temperaturas máximas y mínimas (estaciones Comitán y Santa Elena) (Cuadro 1, Fig. 1) con los índices de la cronología estándar de ancho de anillo, madera temprana y madera tardía. La información climática de estas estaciones se tomó del programa Extractor Rápido de Información Climática (ERIC III) (IMTA, 2009).

En el programa Excel, se corrieron análisis de correlación de Pearson entre la cronología estándar de anillo total, madera temprana y tardía con las variables climáticas. Se definieron los meses con mayor correlación y el periodo estacional que más influye en el crecimiento de $P$. oocarpa. Estos análisis se verificaron y validaron estadísticamente, utilizando el programa STATISTICA Kernel Release 5.5 (Stat Soft Inc., 2000). Finalmente, con este mismo programa, se corrieron modelos de regresión entre las variables climáticas (precipitación, temperatura máxima y mínima) y los índices de anillo, con el fin de definir el potencial de la especie para reconstruir variables climáticas

CUADRO 1

Estaciones meteorológicas empleadas para el análisis dendroclimátologico de Pinus oocarpa, en el Ejido Ojo de Agua, La Independencia, Chiapas

TABLE 1

Climatological stations used for the dendroclimate analysis of Pinus oocarpa, at the Ejido Ojo de Agua, La Independencia, Chiapas

\begin{tabular}{|c|c|c|c|c|c|}
\hline \multicolumn{2}{|c|}{ Estaciones climáticas } & \multirow{2}{*}{ Longitud (Oeste) } & \multirow{2}{*}{ Latitud (Norte) } & \multirow{2}{*}{ Altitud (msnm) } & \multirow{2}{*}{ Periodo de datos } \\
\hline Lugar & Municipio & & & & \\
\hline Comitán & Comitán & 92.117 & 16.25 & 1596 & $1961-2004$ \\
\hline Margaritas & Margaritas & 91.975 & 16.311 & 1512 & $1962-2004$ \\
\hline Santa Elena & Las Margaritas & 91.967 & 16.317 & 560 & $1966-1990$ \\
\hline Tziscao & Trinitaria & 91.633 & 16.1 & 1475 & $1977-1996$ \\
\hline
\end{tabular}


y determinar aquellas que mejor explicaran la variabilidad interanual.

\section{RESULTADOS}

Anillos de crecimiento de $P$. oocarpa: De un total de 65 muestras de 34 árboles de $P$. oocarpa, después de un riguroso reconocimiento de cada anillo e identificación de problemas de crecimiento (anillos falsos y ausentes), se lograron fechar 30 muestras $(46 \%)$ de 22 árboles. El resto de las muestras (54\%) no se logró fechar por presentar alto porcentaje de anillos falsos y períodos de supresión. Para las 30 muestras datadas, se determinó un alto porcentaje de anillos falsos (8 a $60 \%$ ); donde 1991 y 1997 sobresalieron con 57 \% y $52 \%$, respectivamente (Fig. 2).

Los años 1981, 1984, 1985, 1997 y 1999 , son un ejemplo de los anillos falsos que registra el crecimiento de $P$. oocarpa en esta región del trópico mexicano (Fig. 3). Sin embargo, algunas muestras para años específicos no registran problemas de formación de anillos falsos, un ejemplo es el año 1994, que representa un crecimiento normal, condición atípica en la especie para esta región del país (Fig. 3). A pesar de los problemas de crecimiento que presenta $P$. oocarpa y que dificulta su fechado, se logró determinar un patrón de crecimiento entre muestras. Los resultados de COFECHA indicaron una correlación altamente significativa entre series $(r=0.50 ; \mathrm{P}<0.01)$ (Cuadro 2 ). Se lograron generar cronologías de anillo total, madera temprana y madera tardía para los últimos 91 años (1925-2015).

Respuesta del crecimiento a la precipitación: Los análisis de correlación entre la cronología estándar y los datos climáticos de la precipitación regional (1961-2004), indican que la precipitación promedio mensual presenta correlaciones positivas con las cronologías de anillo total, madera temprana y madera tardía, excepto el mes julio, que registró una relación negativa con la cronología de madera tardía ( $\mathrm{r}=$-0.0071) (Fig. 4). Los meses de marzo, abril, mayo, noviembre y diciembre, registran correlaciones significativas $(\mathrm{p}<0.05)$ con las cronologías de anillo total y de madera temprana de P. oocarpa, y los meses de abril, mayo y diciembre con la cronología de madera tardía ( $\mathrm{p}<0.05$; Fig. 4).

Al considerar la precipitación acumulada, se observan correlaciones significativas $(\mathrm{P}<$ $0.05)$ para las cronologías de anillo total, madera

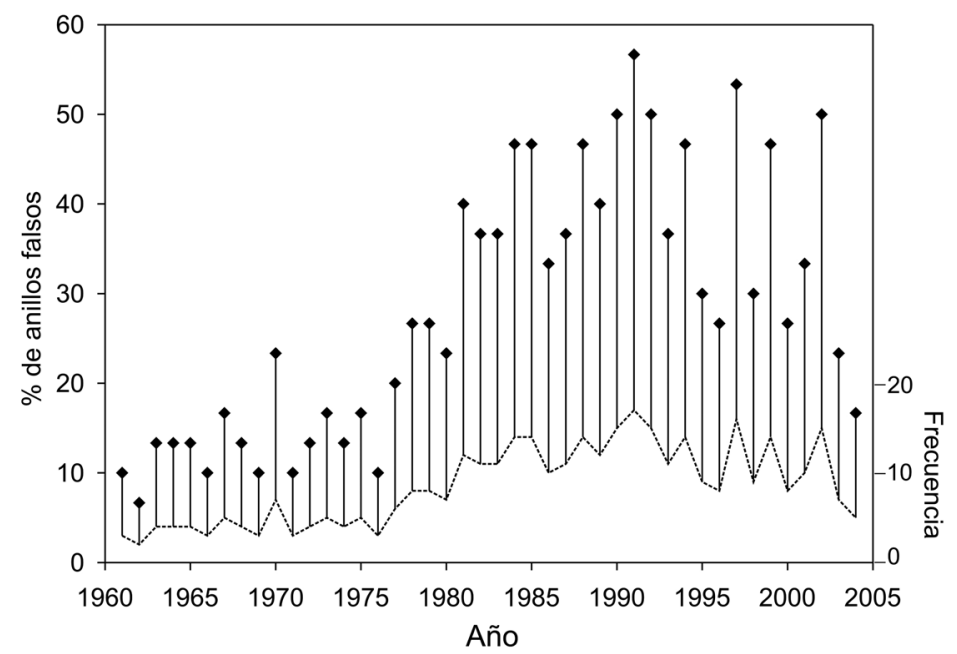

Fig. 2. Porcentaje de anillos falsos en la mayoría de los años de crecimiento de Pinus oocarpa. El menor porcentaje de anillos falsos al inició del gráfico, obedece al tamaño de muestra y no a una menor formación de anillos falsos.

Fig. 2. False ring percentages in the majority of Pinus oocarpa growth years. The lowest percentage at the beginning of the figure is due to the sample depth and not to lower ring false formation. 


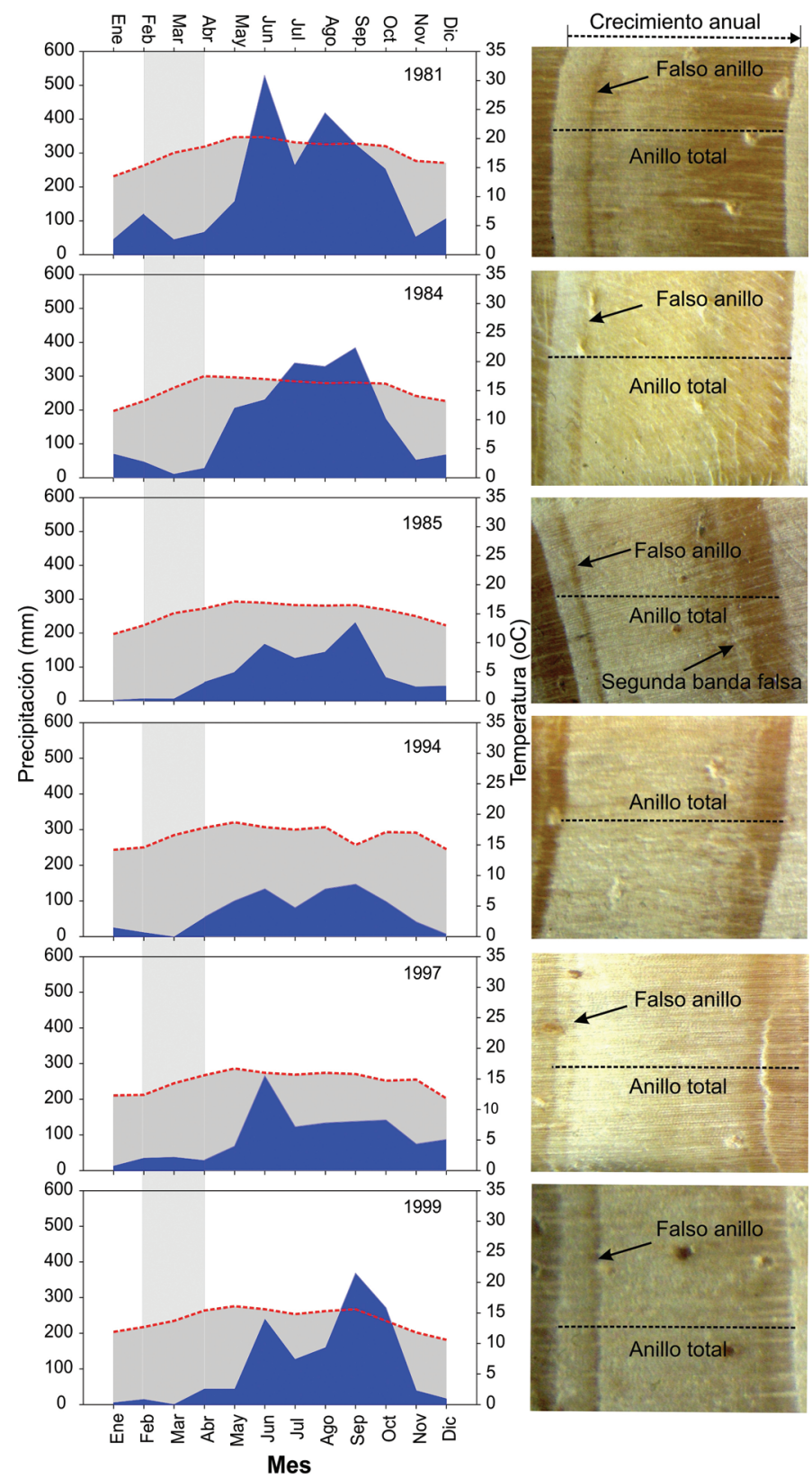

Fig. 3. Climogramas de precipitación y temparatura para diferentes años (izquierda) y crecimientos anuales de Pinus oocarpa (derecha), que muestran la formación característica de anillos falsos en la mayoría de los años como respuesta a una disminución en las condiciones de humedad e incremento en temperatura al inicio de la estación de crecimiento (derecha). La barra en color gris indica el período febrero-marzo con constante disminución en la precipitación e incremento en la temperatura.

Fig. 3. Precipitation and temperature climograms for different years (left) and annual growths of Pinus oocarpa (right) that show the characteristic false rings formation in the most years as a response to a decrease in humidity conditions and an increase in temperature at the beginning of the growing season (right). The bar in gray indicates the period February-March with constant decrease in precipitation and increase in temperature. 
CUADRO 2

Parámetros estadísticos de cronologías desarrolladas con base en crecimientos de Pinus oocarpa

TABLE 2

Statistical parameters of chronologies developed based on annual ring growths of Pinus oocarpa

\begin{tabular}{lcccccc}
\multicolumn{1}{c}{ Sitio } & No. árboles & No. radios & Longitud de la serie & Correlación $^{\mathrm{a}}(\mathrm{r})$ & Sensibilidad $^{\mathrm{b}}$ & Autor \\
RBSM, Guatemala & 21 & 32 & $1880-2005$ & 0.57 & 0.36 & Cerano et al. (2008) \\
Pedernal, Guatemala & 7 & 18 & $1811-2010$ & 0.44 & 0.23 & Szejner (2011) \\
La Primavera, Jalisco & 41 & 35 & $1850-2014$ & 0.50 & 0.32 & Villanueva et al. (2018) \\
Ojo de Agua, Chiapas & $\mathbf{2 2}$ & $\mathbf{3 0}$ & $\mathbf{1 9 2 5 - 2 0 1 5}$ & $\mathbf{0 . 5 0}$ & $\mathbf{0 . 2 8}$ & Trabajo actual \\
\hline
\end{tabular}

RBSM: Reserva de la Biosfera Sierra las Minas.

a Representa la correlación promedio entre todas las series de crecimiento y la cronología promedio.

${ }^{\mathrm{b}}$ La Sensibilidad media determina la diferencia relativa en el ancho de los anillos de un año al siguiente.

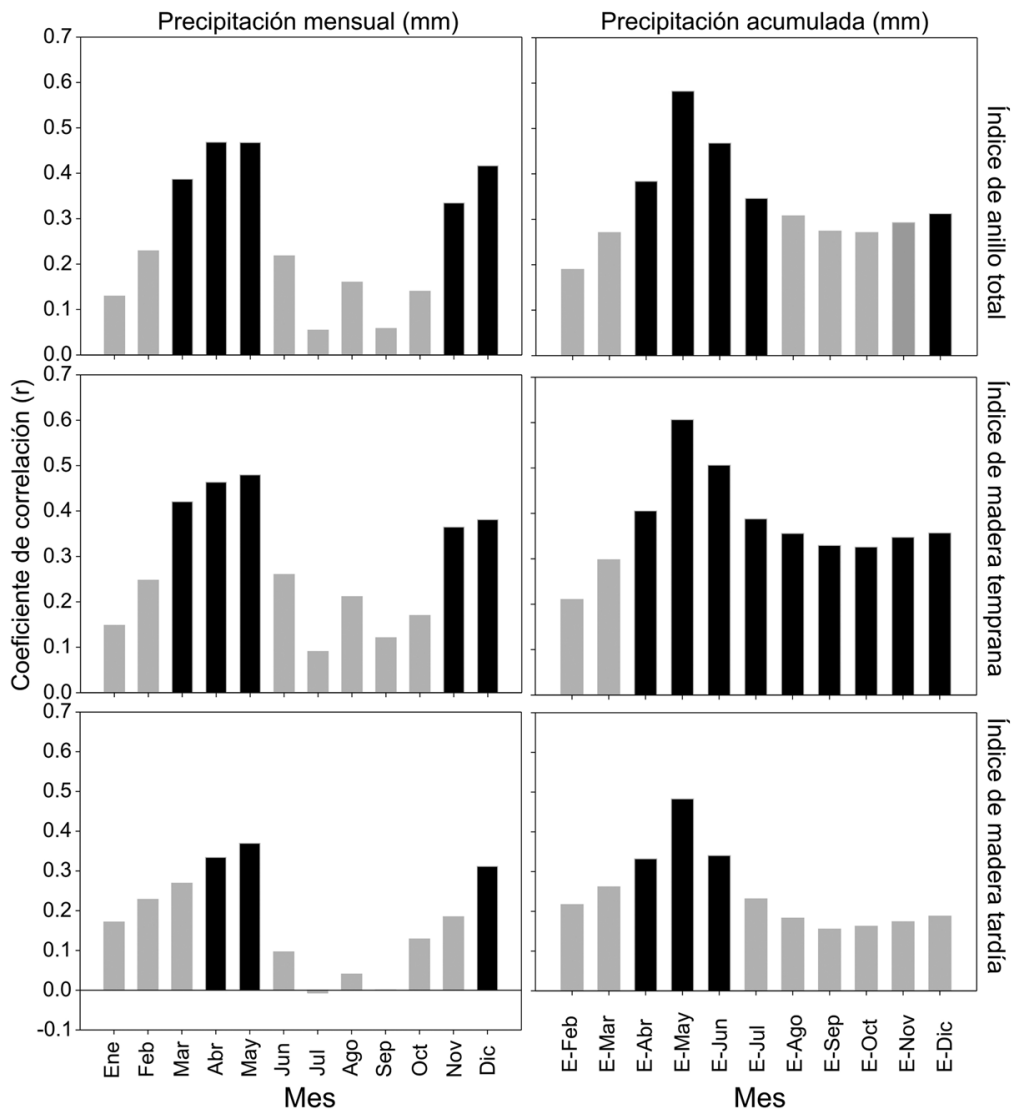

Fig. 4. Coeficiente de correlación entre la precipitación promedio mensual y acumulada del período 1961 a 2004 y los índices de anillo total, madera temprana y madera tardía de Pinus oocarpa. Las barras en color negro representan una correlación significativa $(\mathrm{P}<0.05)$.

Fig. 4. Correlation coefficient between monthly average precipitation and accumulative from 1961 to 2004, and total annual ring, earlywood and latewood indexes of Pinus oocarpa. The black bars represent significant correlations $(\mathrm{P}<0.05)$. 
temprana, y madera tardía con la precipitación enero-abril, enero-mayo y enero-junio. Sin embargo, la mayor correlación se determinó con el período enero-mayo para las tres cronologías (Fig. 4). La cronología de madera temprana registra la más alta correlación con la lluvia estacional enero-mayo (invierno-primavera) (Fig. 4).

Al relacionar únicamente los índices de anillo con la precipitación acumulada de los meses con mayor correlación (marzo-mayo), se observó la mayor relación significativa $(\mathrm{P}<$ $0.05)$ con las cronologías de anillo total, madera temprana y madera tardía, con asociaciones de $0.70,0.71$ y 0.53 , respectivamente (Fig. 5). Correlaciones que superan a las observadas para el período enero-mayo. El análisis de regresión indica potencial para reconstruir la variabilidad de la precipitación de la estación de primavera, considerando como variable independiente la cronología de madera temprana, serie que registra el mayor coeficiente de correlación y por lo tanto explica la mayor variabilidad de la lluvia estacional (Cuadro 3, Fig. 6).

Respuesta del crecimiento a la temperatura: La relación entre las cronologías de anillo total, madera temprana y tardía con los datos climáticos promedio mensual de temperatura mínima y máxima, indicó que la temperatura mínima mensual, presenta correlaciones positivas con las cronologías de anillo total, madera temprana y madera tardía (Fig. 7).

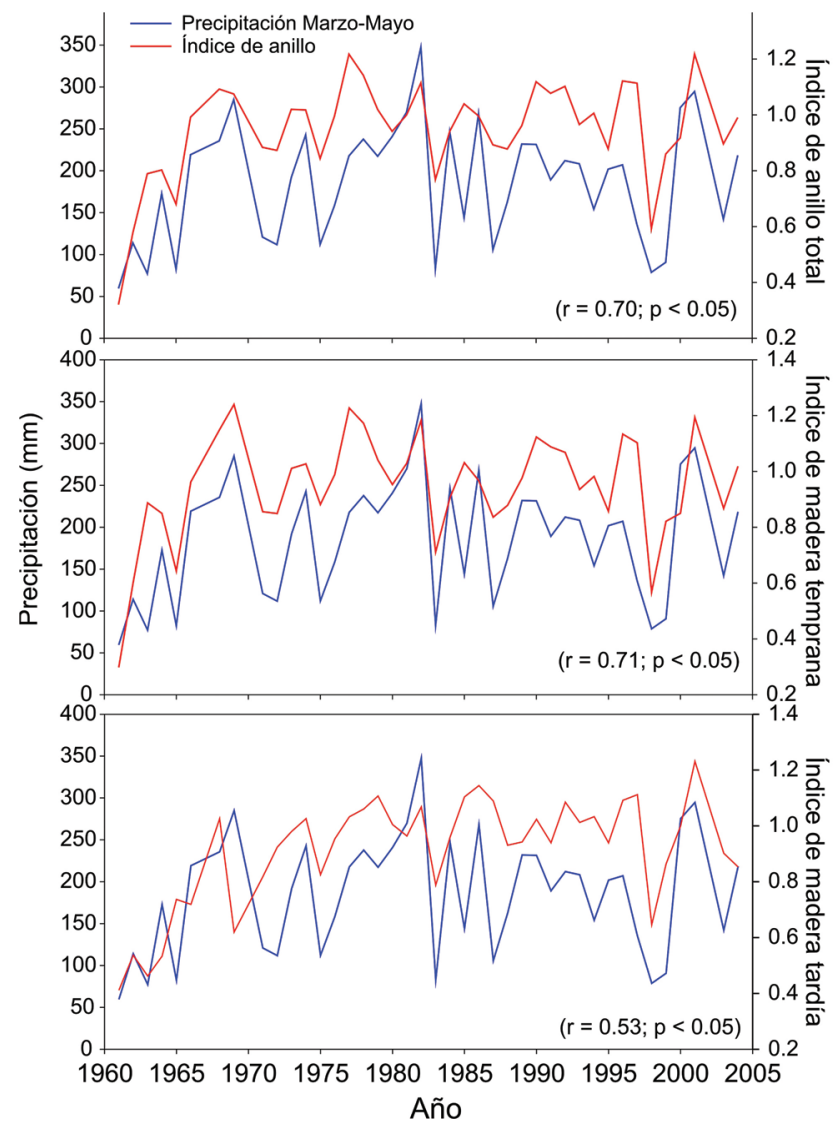

Fig. 5. Relación entre la precipitación estacional marzo-mayo y el índice de anillo total, madera temprana y madera tardía de Pinus oocarpa.

Fig. 5. Relationship between March-May seasonal precipitation and total annual ring width, earlywood and latewood indexes of Pinus oocarpa. 

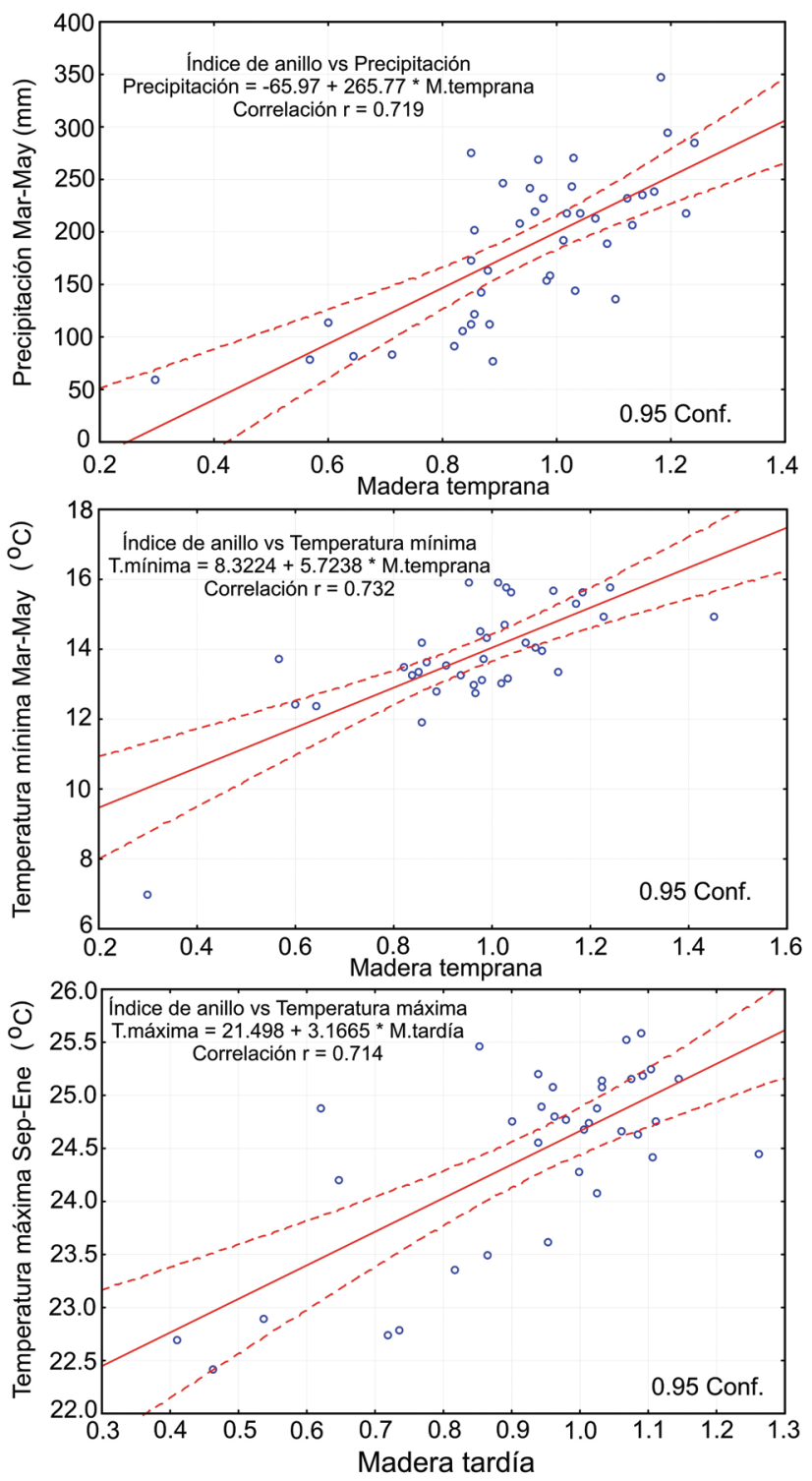

Fig. 6. Diagramas de dispersión de los mejores modelos de regresión entre variables climáticas y las cronologías de madera temprana y madera tardía.

Fig. 6. Scatter plots for the best regression models for climate variables and earlywood and latewood chronologies.

La temperatura mínima registra correlaciones significativas $(\mathrm{P}<0.05)$ con las cronologías de anillo total y madera temprana para todos los meses del año, mientras que la cronología de madera tardía, presenta correlación significativa $(\mathrm{P}<0.05)$ para los meses de febrero, marzo, abril, mayo, noviembre y diciembre (Fig. 7).
Al considerar la temperatura mínima promedio por períodos, se determinó que todos los meses presentan correlaciones significativas $(\mathrm{P}<0.05)$ con las cronologías de anillo total, madera temprana y madera tardía. Sin embargo, el periodo estacional marzo-mayo es el que influye de manera más importante $(\mathrm{P}<0.05)$ 
CUADRO 3

Modelos de regresión lineal que indican la relación significativa $(\mathrm{P}<0.05)$ de los índices de crecimiento con las variables climáticas

TABLE 3

Lineal regression models that indicate a significant relationship $(\mathrm{P}<0.05)$ between ring growth indexes and climate variables

\begin{tabular}{|c|c|c|c|c|c|c|}
\hline Variable climática & Modelo & Parámetros estimados & Error estándar & Cuadrado medio & $\mathrm{R}^{2}$ & $\operatorname{Pr}>F$ \\
\hline \multirow[t]{6}{*}{ Precipitación } & Intercepto & -78.2108 & 43.61544 & 98467.50 & 0.49 & 0.080 \\
\hline & Anillo Total & 278.8658 & 45.38311 & & & $<0.000$ \\
\hline & Intercepto & -65.9745 & 39.62307 & 103576.6 & 0.52 & 0.103 \\
\hline & M. Temprana & 265.7699 & 41.09875 & & & $<0.000$ \\
\hline & Intercepto & 9.1847 & 46.08078 & 56267.53 & 0.28 & 0.843 \\
\hline & M. Tardía & 192.5270 & 49.30295 & & & $<0.000$ \\
\hline \multirow[t]{6}{*}{ T. mínima } & Intercepto & 8.112183 & 1.010391 & 46.1967 & 0.50 & $<0.000$ \\
\hline & Anillo Total & 5.939023 & 1.028413 & & & $<0.000$ \\
\hline & Intercepto & 8.322355 & 0.899073 & 50.0373 & 0.54 & $<0.000$ \\
\hline & M. Temprana & 5.723840 & 0.912698 & & & $<0.000$ \\
\hline & Intercepto & 9.757985 & 1.143384 & 26.2018 & 0.28 & $<0.000$ \\
\hline & M. Tardía & 4.372182 & 1.199855 & & & $<0.000$ \\
\hline \multirow[t]{6}{*}{ T. máxima } & Intercepto & 21.99617 & 0.702143 & 12.4319 & 0.35 & $<0.000$ \\
\hline & Anillo Total & 3.08091 & 0.714667 & & & $<0.000$ \\
\hline & Intercepto & 22.32631 & 0.665659 & 11.4641 & 0.33 & $<0.000$ \\
\hline & M. Temprana & 2.73975 & 0.675747 & & & $<0.000$ \\
\hline & Intercepto & 21.49784 & 0.507707 & 13.74353 & 0.51 & $<0.000$ \\
\hline & M. Tardía & 3.16651 & 0.532782 & & & $<0.000$ \\
\hline
\end{tabular}

Los parámetros indicados en negritas representan los mejores modelos.

Bold parameters indicate the best models.

en el crecimiento de $P$. oocarpa para las tres cronologías, con valores de $0.70,0.73$ y 0.53 , respectivamente (Fig. 8). La mayor correlación se obtuvo con la cronología de madera temprana, por lo que se consideró como variable independiente en el modelo de regresión con fines de reconstrucción de esta variable (Cuadro 3, Fig. 6).

Para el caso de la temperatura máxima mensual, se determinó una relación significativa $(\mathrm{p}<0.05)$ con las cronologías de anillo total, madera temprana y madera tardía de forma consistente para los meses de julio, septiembre y octubre (Fig. 7). Las correlaciones entre la temperatura máxima con las cronologías de anillo total y madera temprana para los meses de enero-mayo y diciembre no son significativas $(\mathrm{P}>0.05)$, por el contrario, para algunos meses (febrero y marzo) son negativas. En contraste, la cronología de madera tardía presentan correlaciones significativas ( $\mathrm{P}$ $<0.05$ ), con los meses de enero y marzo, y las mayores correlaciones $(\mathrm{P}<0.05)$ se registran en noviembre y diciembre.

Al relacionar la temperatura máxima promedio de septiembre y octubre con las cronologías de anillo total y madera temprana, se determinó una relación significativa $(\mathrm{P}<0.05)$ de 0.59 y 0.57 , respectivamente (Fig. 8). Sin embargo, la mayor correlación se observó entre la temperatura máxima septiembre-enero y la cronología de madera tardía $\mathrm{r}=0.71(\mathrm{P}<0.05)$ (Fig. 8). Existe una importante influencia de la temperatura en el crecimiento de $P$. oocarpa en 

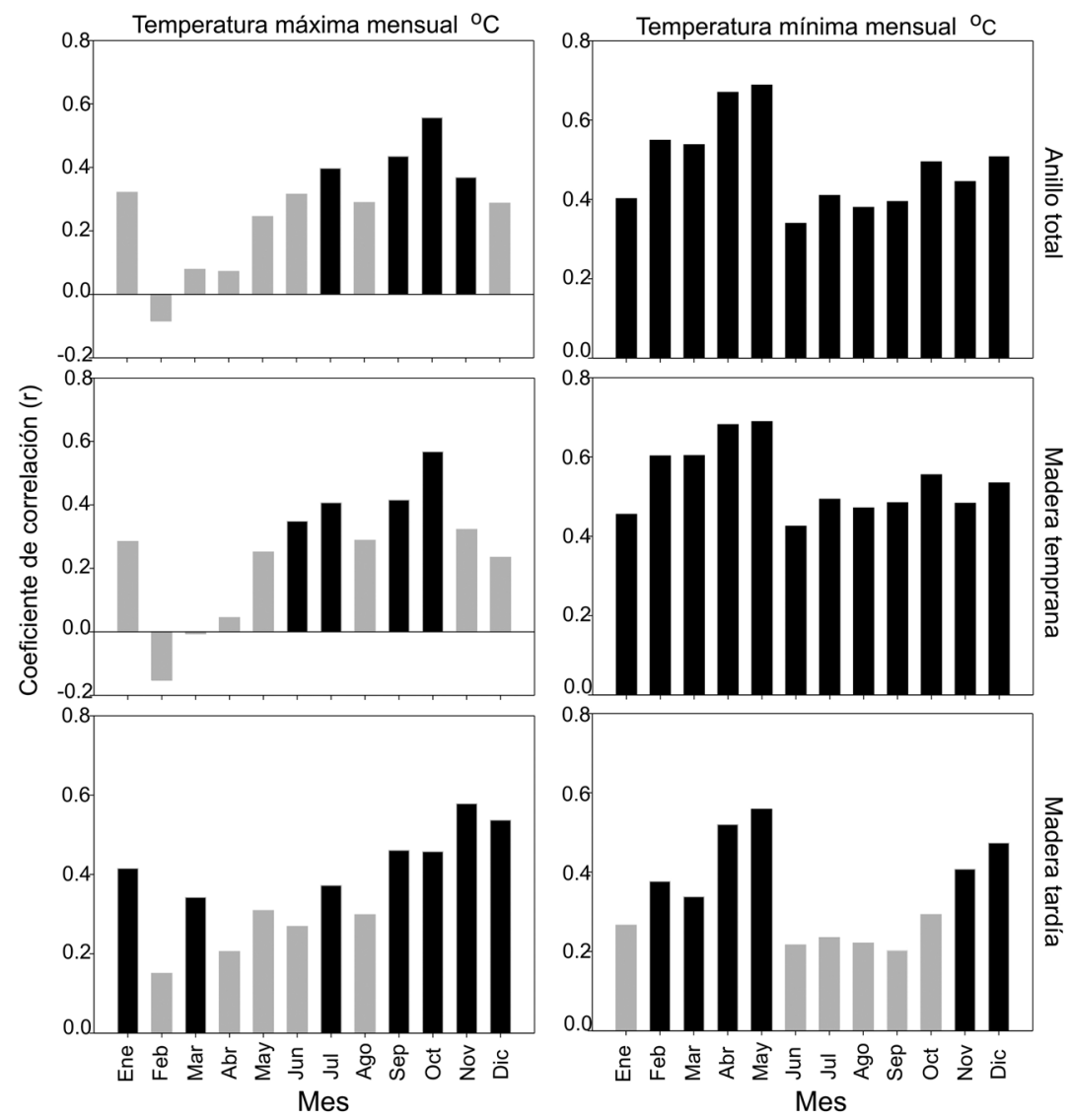

Fig. 7. Coeficiente de correlación entre la temperatura promedio máxima y mínima mensual (1961 a 2004) y el índice de anillo total, madera temprana y madera tardía de Pinus oocarpa. Las barras en color negro representan una correlación significativa $(\mathrm{P}<0.05)$.

Fig. 7. Correlation coefficients between monthly average minimum and maximum temperature from 1961 to 2004 , and total annual ring width, earlywood and latewood indexes of Pinus oocarpa. Black bars represent a significant correlation $(\mathrm{P}<0.05)$.

esta región de Chiapas. La temperatura mínima juega un papel importante en el crecimiento de la madera temprana y la temperatura máxima en el desarrollo de la madera tardía. Las cronologías generadas con base en los anillos de crecimiento de $P$. oocarpa presentan potencial para reconstruir la temperatura mínima marzomayo con base en la cronología de madera temprana y la temperatura máxima septiembreenero con base en la cronología de madera tardía (Cuadro 3, Fig. 6).

\section{DISCUSIÓN}

Anillos de crecimiento de $P$. oocarpa: Los anillos de crecimiento anual de los árboles están influenciado por varios factores ambientales que interactúan entre sí, como radiación solar, temperatura, precipitación, contenido de nutrientes del suelo, entre otros. Dependiendo de las condiciones ambientales y de las especies, uno o más de estos factores pueden limitar el crecimiento (Fritts, 1976; Bogino 

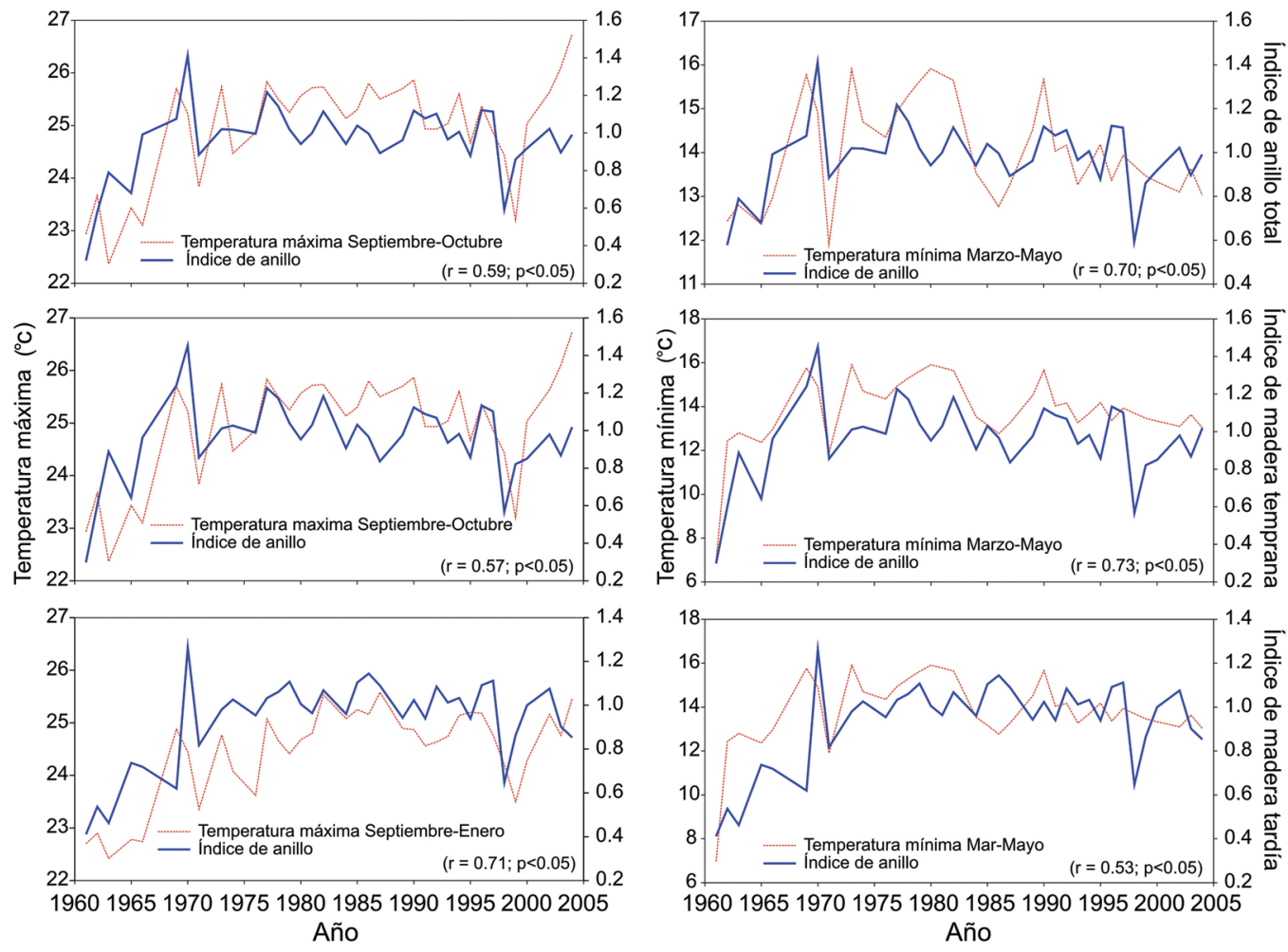

Fig. 8. Gráficos que muestran las mejores correlaciones entre la temperatura mínima estacional marzo-mayo con el índice de anillo total, madera temprana y madera tardía. Así mismo, la temperatura máxima estacional septiembre-octubre con el anillo total y la madera temprana y septiembre-enero con el índice de la madera tardía.

Fig. 8. Best correlations between seasonal minimum temperature, from March to May, and the total annual ring, earlywood and latewood indexes, as well as, between seasonal maximum temperature, from September to October, and the total annual ring and earlywood; and between seasonal maximum temperatures, from September to January, with the latewood index.

\& Bravo, 2008). El crecimiento radial de $P$. oocarpa muestra estructuras anatómicas como madera temprana y madera tardía; así mismo, anomalías en la densidad de la madera conocidas como anillos falsos que pueden relacionarse con eventos hidro-meteorológicos (Fig. 3). Por ejemplo, la disponibilidad de humedad invernal, favorece el crecimiento inicial de $P$. oocarpa (Fig. 4), una disminución de humedad y altas temperaturas durante los meses de febrero y marzo propician un cese en la actividad cambial del árbol, dando paso a la formación de una banda de crecimiento falsa (Fig. 3). Como lo sugiere Vaganov, Hughes y Shashkin (2006), la actividad secundaria del crecimiento de P. oocarpa en el área de estudio parece desencadenarse con las lluvias que se presentan al final de la primavera e inicio del verano. La Zona de Convergencia Intertropical (ZCIT) está relacionada con el inicio de la temporada de lluvias en estas latitudes, produciendo precipitaciones durante el verano (Curtis, 2002). Esta condición está asociada de manera positiva con el crecimiento de la madera temprana de $P$. oocarpa (Fig. 4), caracterizada por grandes traqueidas, paredes celulares delgadas y gran lumen.

Para realizar un análisis dendroclimático, la identificación correcta del inicio y final del anillo de crecimiento anual es un paso fundamental para lograr una datación confiable (Stokes \& Smiley, 1996; Stahle, 1999). La comparación de los patrones de crecimiento con base en el fechado cruzado empleando 
gráficos de crecimiento o "skeleton plots", permitieron identificar y discriminar problemas de crecimiento. Esta técnica trata no solo de la sincronización, es útil para reconocer anillos falsos, perdidos y permite corregir y asignar el año exacto a cada anillo, es el principio más importante en dendrocronología (Fritts, 1976; Cook, 1985; Stokes \& Smiley, 1996). La datación cruzada permite estar seguros sobre la fecha de cada anillo de crecimiento en muestras diferentes. Adicionalmente, se empleó el programa COFECHA que considera la posible existencia de anillos falsos mediante la falta de una buena correlación entre muestras (Holmes, 1983).

La dificultad para lograr un fechado correcto de los crecimientos anuales de $P$. oocarpa atribuido a la formación de anillos falsos y períodos de supresión, ha sido reportado en estudios dendroclimáticos desarrollados en Centroamérica (Hastenrath, 1963; Szejner, 2011). El fechado de $46 \%$ de las muestras de $P$. oocarpa en este estudio, corrobora lo planteado por Villalba (1990), Lara y Villalba (1993) y Neira (1995), quienes han documentado, que el porcentaje de muestras fechadas, puede variar de 43 a $90 \%$.

A pesar de los problemas de crecimiento que presenta $P$. oocarpa, los resultados de COFECHA indicaron una correlación significativa entre series $(\mathrm{r}=0.50 ; \mathrm{P}<0.01)$, superando los parámetros estadísticos que dicho programa establece $(\mathrm{r}=0.3281, \mathrm{P}<0.01)$ (Holmes, 1983). Los parámetros estadísticos de esta serie son similares a los reportados para $P$. oocarpa en el bosque La Primavera, Jalisco (Villanueva et al., 2018) y la Reserva de la Biosfera Sierra de las Minas (RBSM) en Guatemala (Cerano et al., 2008); pero superiores a los parámetros determinados por Szejner (2011) en el sitio Pedernal en Guatemala (Cuadro 2). Estos resultados indican la sensibilidad de $P$. oocarpa a los cambios ambientales y su potencial para emplearse en el desarrollo de reconstrucciones dendroclimáticas (Fritts, 1976; Delgado, 2000; Grissino-Mayer, 2001). Se logró generar una cronologia para los últimos 91 años (19252015) y con base en estos resultados, se cubre el primer objetivo y se da respuesta a la primera pregunta de investigación.

Respuesta del crecimiento a la precipitación: Los análisis de correlación entre la cronología y los datos climáticos de la precipitación regional (1961-2004), indican que la precipitación presenta correlaciones positivas con el crecimiento de $P$. oocarpa. Sin embargo, los meses del período estacional inviernoprimavera indican una mayor influencia en el crecimiento. Una respuesta similar del crecimiento de $P$. oocarpa a la lluvia estacional invierno-primavera, se reportó para el bosque La Primavera en Jalisco (Villanueva et al., 2018). Sin embargo, la respuesta de $P$. oocarpa es diferente en regiones de Guatemala, donde la mayor influencia en crecimiento ocurre con la precipitación primavera-verano (Cerano et al., 2008; Szejner, 2011).

A pesar de la amplia distribución de esta especie son pocos los trabajos que han estudiado la influencia del clima en su crecimiento. Hastenrath (1963) reportó el primer estudio de anillos en $P$. oocarpa en El Salvador, en la búsqueda de relacionar la variabilidad de la lluvia y el crecimiento, encontró una relación positiva entre ambas variables. Johnson (1980) en Copan, Honduras, estudió la anatomía del anillo anual para entender los factores ambientales que influyen en el crecimiento de los árboles. Cerano et al. (2008) en un estudio en Sierra Las Minas, Guatemala, determinaron una influencia significativa de la precipitación mayo-septiembre en el crecimiento de $P$. oocarpa. Szejner (2011) al este de Guatemala relacionó el crecimiento de $P$. oocarpa con la precipitación reportando que esta especie responde de manera significativa $(\mathrm{P}<0.001)$ al periodo mayo-julio (primavera-verano); mientras que para los Tuxtlas, Veracruz, México, Gutiérrez (2013) señala una influencia de la precipitación diciembre-junio en el crecimiento de la madera total de esta especie.

A diferencia de la respuesta del crecimiento de $P$. oocarpa a la lluvia primavera-verano en Centroamérica (Cerano et al., 2008; Szejner, 2011), en el trópico mexicano esta especie 
responde a la lluvia estacional invierno-primavera similar a otras coníferas distribuidas en el norte, centro y sur de México (Cleaveland et al., 2003; Constante, Villanueva, Cerano, Cornejo, \& Valencia, 2009; Villanueva et al., 2009; Santillán et al., 2010; Cerano et al., 2011, 2013, 2014).

Respuesta del crecimiento a la temperatura: La relación entre las cronologías y las temperaturas, indican una influencia significativa tanto de la temperatura máxima como mínima en el crecimiento de $P$. oocarpa. La temperatura mínima de primavera (marzomayo) es el período con mayor influencia ( $\mathrm{P}<$ 0.05 ) en el crecimiento de $P$. oocarpa, lo cual se atribuye, a que, en este período, la temperatura mínima se ubica en un rango que permite la actividad fotosintética, ya que temperaturas cercanas a $5{ }^{\circ} \mathrm{C}$ o por debajo de ésta, propician que los estomas se cierren interrumpiendo el intercambio de gases (Barceló, Nicolás, Sabater, \& Sánchez, 2001). Por otra parte, el proceso de evapotranspiración se incrementa con mayores temperaturas abatiendo en menor tiempo la disponibilidad hídrica en el suelo y en consecuencia se reduce el crecimiento.

Las correlaciones significativas $(\mathrm{P}<0.05)$ de noviembre, diciembre, enero y marzo con la cronología de madera tardía, contrario a lo observado para la madera temprana, puede explicar la formación de anillos falsos al inicio del crecimiento (Fig. 3). Esto se puede atribuir a que al registrarse temperaturas por encima de $25{ }^{\circ} \mathrm{C}$ producen una fuerte presión al nivel de los estomas, el déficit de presión de vapor se incrementa hasta por arriba de los $2.0 \mathrm{kPa}$, lo que provoca cierre de estomas y se inhibe el intercambio de gases y en consecuencia el crecimiento radial (Barceló et al., 2001). Esto produce la acumulación de carbohidratos en las paredes de las células aumentando su espesor (Bidwell, 1979; Giménez, Moglia, Hernández, \& Gerez, 2014). La menor correlación del crecimiento temprano con la temperatura máxima, significa que, a una mayor temperatura al inicio de la estación de crecimiento se favorece un menor crecimiento del anillo de $P$. oocarpa, caso contrario a la respuesta con la temperatura mínima.

Una mayor respuesta del crecimiento temprano a la temperatura mínima y menor relación ante condiciones de mayor temperatura, ha sido documentada en diversos estudios. Huante, Rincón y Swetnam (1991) reportan una correlación significativa del incremento radial anual de Abies religiosa con la temperatura media y mínima de enero y febrero. Cerano et al. (2014) encontraron una relación significativa $(\mathrm{P}<0.05)$ de la temperatura media y mínima de enero y febrero con el crecimiento anual de Abies religiosa. Cerano, Rivera, Estrada, Trucios y Ríos (2012) documentaron que los crecimientos de la madera temprana de Pinus cooperi, responde de manera significativa $(\mathrm{P}>0.05)$ a la temperatura mínima de enero, febrero, marzo y abril. Un estudio reciente ha reportado una influencia significativa $(\mathrm{P}<0.05)$ de la temperatura mínima de enero y febrero en el crecimiento de Pinus cooperi y Pinus durangensis, y una respuesta negativa con la temperatura máxima (Chávez et al., 2017).

Las cronologías generadas con base en los anillos de crecimiento de $P$. oocarpa tienen potencial como una fuente "proxy" para reconstruir variables climáticas, como la precipitación y la temperatura mínima de primavera (marzo-mayo) con base a la cronología de madera temprana, y temperatura máxima septiembre-enero con base en la cronología de madera tardía. Estos resultados cubren el segundo objetivo de esta investigación y sustentan la segunda pregunta de investigación.

Esta especie representa una de las primeras coníferas en México en registrar altas correlaciones con la variabilidad de las temperaturas. Estos resultados son de gran relevancia en dos aspectos: 1) $P$. oocarpa representa una de las pocas coníferas con amplia distribución en esta región, por lo tanto, se podrán generar registros extensos en diferentes áreas del trópico, y 2) que, ante el incremento de la temperatura, esta especie constituye una opción potencial para su reconstrucción, analizar su variabilidad interanual por décadas o siglos y analizar posibles 
tendencias ante los diversos escenarios de cambio climático. Es importante dar continuidad a este tipo de estudios, detectar áreas con arbolado longevo y lograr generar series extensas que cubran al menos los últimos dos siglos, con el objetivo de analizar tendencias y recurrencia de eventos hidro-climáticos extremos. Este trabajo de investigación aporta conocimiento que contribuye a fomentar los estudios dendroclimáticos en esta región del trópico mexicano.

Declaración de ética: los autores declaran que todos están de acuerdo con esta publicación y que han hecho aportes que justifican su autoría; que no hay conflicto de interés de cualquier tipo; y que han cumplido con todos los requisitos y procedimientos éticos y legales pertinentes. El documento firmado se encuentra en los archivos de la revista.

\section{AGRADECIMIENTOS}

Un agradecimiento especial a Roberto García Cancino por su apoyo en la logística durante el muestreo de campo, al ejido Ojo de Agua, La Independencia, Chiapas, por permitirnos el acceso y la toma de muestras. Así mismo, gracias a Gerardo López, José Luis Hernández, Adolfo Cruz, Valdemar Guillen y Maynor Morales por el apoyo en el levantamiento de datos de campo. Este proyecto fue financiado con fondos personales de los autores.

\section{RESUMEN}

Los estudios dendrocronológicos se utilizan para reconstruir algunas variables climáticas; en México, estos estudios se han centrado en los bosques templados del centro y del norte, donde los árboles presentan anillos anuales bien definidos. Pocos estudios se han llevado a cabo en la parte sur del país, donde el crecimiento anual del anillo no se identifica fácilmente $y$, por lo tanto, esto hace que la datación se dificulte. Se analizó el potencial dendrocronológico de Pinus oocarpa para la reconstrucción de variables climáticas en la porción noroeste de Lagunas de Montebello, Chiapas. Empleando un muestreo selectivo se recolectaron 65 núcleos de incremento de 34 árboles. Aunque las muestras presentaron una alta frecuencia de anillos falsos ( 8 a $60 \%$ ), se logró fechar 30 muestras de 22 árboles (46\%) mediante técnicas dendrocronológicas estándar y el desarrollo de cronologías de anillo total, madera temprana y madera tardía para un período de 91 años (1925-2015). Se encontró una influencia significativa de la precipitación media y de la temperatura media máxima y mínima del período 1961-2004 sobre el crecimiento anual de $P$. oocarpa. Los resultados muestran que la precipitación inviernoprimavera (enero-mayo) fue la más importante para el crecimiento del anillo anual de la especie. Sin embargo, la correlación más alta se observó entre la precipitación de primavera (marzo-mayo) y la cronología de la madera temprana $(\mathrm{r}=0.719, \mathrm{P}<0.05)$. La cronología de la madera temprana también mostró potencial para reconstruir la temperatura mínima (marzo a mayo) $(\mathrm{r}=0.732, \mathrm{P}<0.05)$, mientras que la cronología de madera tardía registra potencial para reconstruir la temperatura máxima (septiembreenero) $(\mathrm{r}=0.714, \mathrm{P}<0.05)$. Estos resultados muestran que $P$. oocarpa puede emplearse para reconstruir variables climáticas en los trópicos mexicanos. Se recomienda explorar nuevas áreas con árboles más viejos a fin de aumentar la extensión de las cronologías y reconstruir los registros climáticos varios siglos en el pasado.

Palabras claves: anillos de crecimiento; anillos falsos; madera temprana; madera tardía; potencial dendroclimático.

\section{REFERENCIAS}

Barceló, J., Nicolás, G., Sabater, B., \& Sánchez, R. (2001). Fisiología vegetal. Madrid, España: Ediciones Pirámide.

Benito, F. G. (2014). Archivos climáticos y paleohidrológicos. Introducción a datos proxy y su análisis. Madrid, España: CSIC- Museo Nacional de Ciencias Naturales.

Bidwell, R. G. S. (1979). Fisiología vegetal. México: AGT Editor.

Bogino, S. M., \& Bravo, F. (2008). Growth response of Pinus pinaster Ait. to climatic variables in central Spanish forests. Annals of Forest Science, 65(5), 506-506.

Bradley, R. S. (1999). Paleoclimatology: Reconstructing climates of the Quaternary. ( $2^{\text {nd }}$ ed.). USA: Academic Press.

Cerano, P. J., Méndez, G. J., Amaro, S. A., Villanueva, D. J., Cervantes, M. R., \& Rubio, C. E. (2013). Reconstrucción de precipitación invierno-primavera con anillos anuales de Pinus douglasiana en la Reserva de la Biósfera de Manantlán, Jalisco. Revista Chapingo Serie Ciencias Forestales y del Ambiente, 29(3), 413423. DOI: 10.5154/r.rchscfa.2013.02.007

Cerano, P. J., Rivera, G. M., Estrada, A. J., Trucios, C. R., \& Ríos, S. J. C. (2012). Análisis dendrocronológico de Pinus cooperi en Durango, México. Agrofaz, 12(3), 81-88. 
Cerano, P. J., Villanueva, D. J., Cervantes, M. R., Vázquez, S. L., Trucios, C. R., \& Guerra, C. V. (2014). Reconstrucción de precipitación invierno-primavera para el Parque Nacional Pico de Tancítaro, Michoacán. Investigaciones Geográficas, 83,41-54.

Cerano, P. J., Villanueva, D. J., Moran, M. R., Sheppard, P. R., Bradley, T., \& Kaib, M. (Noviembre, 2008). Reconstrucción histórica de precipitación con anillos de árboles para el Este de Guatemala. In J. J. Martínez-Ríos, M. Vázquez-Navarro, A. Martínez-Ríos, S. Berúmen-Padilla, \& R. Santana-Rodríguez (Eds.), XX Semana Internacional de Agronomía. Facultad de Agricultura y Zootecnia-Universidad Juárez del Estado de Durango, México.

Cerano, P. J., Villanueva, D. J., Valdez, C. R. D., Cornejo, O. E. H., Sánchez, C. I., \& Constante, G. V. (2011). Variabilidad histórica de la precipitación reconstruida con anillos de árboles para el sureste de Coahuila. Revista Mexicana de Ciencias Forestales, 2(4), $31-45$.

Chávez, G. M. P., Cerano, P. J., Nájera, L. J. A., Pereda, B. V., Esquivel, A. G., Cervantes, M. R., ... Corral, R. S. (2017). Reconstrucción de la precipitación inviernoprimavera con base en anillos de crecimiento de árboles para la región de San Dimas, Durango, México. Bosque, 38(2), 387-399.

Cleaveland, M. K., Stahle, D. W., Therrell, M. D., Villanueva-Díaz, J., \& Burs, B. T. (2003). Tree-ring reconstructed winter precipitation and tropical teleconnections in Durango, Mexico. Climatic Change, 59(1), 369-388. DOI: 10.1023/A

Constante, G. V., Villanueva, D. J., Cerano, P. J., Cornejo, O. E. H., \& Valencia, M. S. (2009). Dendrocronología de Pinus cembroides Zucc. y reconstrucción de precipitación estacional para el Sureste de Coahuila. Ciencia Forestal en México, 34(106), 17-39.

Cook, E. R. (1985). A time series approach to Tree-Ring standardization (Doctoral dissertation). University of Arizona, USA

Cook, E. R. (1987). The decomposition of tree-ring series for environmental studies. Tree-Ring Bulletin, 47, $37-59$.

Curtis, S. (2002). Interannual variability of the bimodal distribution of summertime rainfall over Central America and tropical storm activity in the far-eastern Pacific. Climate Research, 22, 141-146.

Delgado, C. S. (2000). Aplicaciones estadísticas en análisis dendrocronológicos. En F. A. Roig (Ed.), Dendrocronología en América Latina (pp. 79-102). Mendoza, Argentina: Editorial de la Universidad Nacional de Cuyo.

Fritts, H. C. (1976). Tree-rings and Climate. London - New York - San Francisco: Academic Press.
García, E. (1998). Modificaciones al sistema de clasificación climática de Köppen. México: Instituto de Geografía Universidad Nacional Autónoma de México.

Giménez, A. M., Moglia, J. G., Hernández, P., \& Gerez, R. (2014). Anatomía de la madera. (2 $2^{\text {da }}$ ed.). Argentina: Universidad Nacional de Santiago del Estero.

Grissino-Mayer, H. D. (2001). Evaluating crossdating accuracy: a manual and tutorial for the computer program COFECHA. Tree-Ring Research, 57(2), 205-221.

Gutiérrez, G. G. (2013). La relación entre crecimiento y clima en árboles tropicales: un estudio dendrocronológico en la selva de los Tuxtlas, Veracruz, México (Tesis Doctoral). UNAM, México.

Hastenrath, S. (1963). Dendrochronologie en El Salvador. Meteorologische Rundschan, 16, 110-113.

Holmes, R. L. (1983). Computer-assited quality control in tree-ring dating and measurement. Tree-Ring Buletin, $43,69-78$

Huante, P., Rincón, E., \& Swetnam, T. W. (1991). Dendrochronology of Abies religiosa in Michoacán, México. Tree-Ring Bulletin, 51, 5-27.

IMTA (Instituto Mexicano de Tecnología del Agua, MX). (2009). Extractor Rápido de Información Climatológica III (ERIC). Morelos, México: Software, Jiutepec.

INEGI (Instituto Nacional de Estadística y Geografía, MX). (2005). Marco estadístico municipal. Datos Geográficos de la Carta Geológica, 1:250000, serie I. La Independencia, Chiapas.

INEGI (Instituto Nacional de Estadística y Geografía, MX). (2007). Carta edafológica de Chiapas. Escala $1: 250000$

INEGI-CONAGUA (Instituto Nacional de Estadística y Geografía-Comisión Nacional del Agua). (2007). Prontuario de la información geográfica municipal de los Estados Unidos Mexicanos. La Independencia, Chiapas. Recuperado de http://www3.inegi.org.mx/ sistemas/mexicocifras.pdf

Johnson, W. C. (1980). Muestreo dendrocronológico de Pinus oocarpa cerca de Copán, Honduras: una nota preliminar. Biotrópica, 12, 315-316.

Lara, A., \& Villalba, R. (1993). A 3,620-year temperature reconstruction from Fitzroya cupressoides tree rings in southern South America. Science, 260, 1104-1106.

Martínez, M. (1992). Los pinos mexicanos (3 ${ }^{\text {era }}$ ed.). México: Editorial Botas.

Neira, E. P. (1995). Desarrollo de cronologías para alerce (Fitzroya cupressoides) en las Cordilleras de la Costa 
$y$ de los Andes (Tesis de Grado). Universidad Austral de Chile, Valdivia, Chile.

Perry, J. P. Jr., Graham, A., \& Richardson, M. D. (1998). The history of pines in México and Central America. In M. D. Richardson (Ed.), Ecology and Biogeography of Pinus (pp.137-149). Cambridge, UK: Cambridge University Press.

Rzedowski, J. (1978). Vegetación de México. México: Limusa.

Santillán, H. M., Cornejo, E. H., Villanueva, D. J., Cerano, P. J., Valencia, M. S., \& Capó, A. M. A. (2010). Potencial dendroclimático de Pinus pinceana Gordon en la Sierra Madre Oriental. Madera y Bosques, 16, 17-30.

Stahle, D. W. (1999). Useful strategies for the development of tropical tree ring chronologies. IAWA Bulletin, 20(3), 249-253.

Stat Soft Inc. (2000). STATISTICA Software, Kernel Release version 5.5 A. 1984-2000. Tulsa, Oklahoma, USA.

Stokes, M. A., \& Smiley, T. L. (1996). An introduction to the tree-ring dating. USA: The University of Arizona Press.

Szejner, S. P. (2011). Tropical dendrochronology: exploring tree-rings of Pinus oocarpa in Eastern Guatemala (Master's thesis). University of Göttingen, Germany.

Tschinkel, H. M. (1966). Annual growth rings in Cordia alliodora. Turrialba, 16, 73-80.
Turner, I. M. (2004). The Ecology of trees in the tropical rain forest. Cambridge, UK.: Cambridge University Press.

Vaganov, E. A., Hughes, M. K., \& Shashkin, A. V. (2006). Growth dynamics of tree rings: an image of the past and future environments. Berlin: Springer-Verlag.

Villalba, R. (1990). Climatic fluctuations in northern Patagonia during the last 1,000 years as inferred from tree-ring records. Quaternary Research, 34, 346-360.

Villanueva, D. J., Fulé, P. Z., Cerano, P. J., Estrada, A. J., \& Sánchez, C. I. (2009). Reconstrucción de la precipitación estacional para el barlovento de la Sierra Madre Occidental. Revista Ciencia Forestal en México, 34(105), 37-69.

Villanueva, D. J., Cerano, P. J., Fulé, P. Z., Cortés, M. C., Vázquez, S. L., Yocom, L. L., \& Ruiz, C. J. A. (2015). Cuatro siglos de variabilidad hidroclimática en el noroeste de Chihuahua, México, reconstruida con anillos de árboles. Investigaciones Geográficas. Boletín del Instituto de Geografia, 87, 141-153.

Villanueva, D. J., Rubio, C. E. A., Chávez, D. A. A., Zavala, A. J. L., Cerano, P. J., \& Martínez, S. A. R. (2018). Respuesta climática de Pinus oocarpa Schiede Ex Schetol en el Bosque La Primavera, Jalisco. Madera y Bosques, 24(1), e2411464. DOI:10.21829/ myb.2018.2411464

Worbes, M. (2002). One hundred of tree-ring research in the tropics - a brief history and outlook to future challenges. Dendrochronologia, 20, 217-231. 